\title{
Herramientas para evaluar la sostenibilidad de las intervenciones urbanas en barrios
}

\author{
Sustainability assessment tools for urban design at \\ neighbourhood scale
}

M. Simón-Rojo ${ }^{(*)}$ y A. Hernández-Aja ${ }^{(* *)}$

\section{RESUMEN}

El empleo en la planificación y en el diseño de intervenciones de mejora urbana, de herramientas que permitan comparar alternativas con criterios de sostenibilidad, contribuiría a afrontar algunos de los retos fundamentales a los que se enfrentan nuestras ciudades. Desde la Unión Europea se aboga por políticas urbanas integrales. Para lograrlas sería recomendable contar con herramientas de evaluación adecuadas, que incorporen los aspectos socioeconómicos y se puedan aplicar en la escala de barrio.

El artículo presenta las conclusiones de la revisión de herramientas para evaluar la sostenibilidad urbana en el contexto europeo. Estas herramientas aportan sobre todo informes, guías, indicadores y buenas prácticas. En algún caso se han desarrollado metodologías y tecnologías específicas. A pesar de la gran cantidad de recursos públicos invertidos, sigue habiendo una carencia de herramientas prácticas, útiles y de código abierto que permitan evaluar la sostenibilidad de las intervenciones en barrios de la ciudad construida.

\section{$113-115$}

Palabras clave: sostenibilidad, planeamiento urbanístico, herramientas de evaluación, escala de barrio, programas de investigación europeos, Quinto Programa Marco, Sexto Programa Marco, código abierto.

\section{SUMMARY}

Integrating assessment tools in the decision making process of urban design may contribute to tackle the defining challenges of sustainability that our cities face. The research works on practical tools to compare alternatives taking into account sustainability criteria that incorporate quantitative as well as qualitative assessment. Effective assessment tools that take into account socioeconomic criteria are required to push forward integrated urban planning policies that can shape interventions at neighbourhood scale.

This paper presents the conclusions after the review of assessment tools available in the European context. These tools are focussed mainly on delivering reports and guidelines, list of indicators and best practices; besides those reports, also some methodologies and specific technologies have been developed. Nevertheless there is a lack of practical, useful and open source tools to assess urban sustainability of urban small scale interventions.

Keywords: sustainability, urban planning, assessment tools, neighbourhood scale, European research programs FP5, FP6, open source. 


\section{INTRODUCCIÓN}

\subsection{Evaluar la sostenibilidad urbana en el contexto europeo}

Desde que en 1991 la Comisión Europea creara el grupo de expertos en medio ambiente urbano, la Unión Europea trabaja para incorporar objetivos ambientales en las políticas urbanas y lograr ciudades sostenibles. En la actualidad se asume que la adopción de políticas urbanas integradas y la intervención sobre la ciudad existente son indispensables para avanzar hacia la sostenibilidad.

Para lograr estos objetivos es recomendable aplicar herramientas y procedimientos adecuados que permitan medir el impacto y la sostenibilidad de las actuaciones. En la Cátedra TEAM de la Universidad de Delft ${ }^{1}$ se inició una investigación para sopesar la pertinencia de desarrollar una herramienta de evaluación de la sostenibilidad de las intervenciones urbanas en barrios.

Esta herramienta estaría dirigida a técnicos municipales, estudios de urbanismo y otras organizaciones implicadas en actuaciones de mejora urbana, en entornos de limitados recursos económicos y de personal. En este sentido, cabe destacar que la inmensa mayoría de los municipios carecen de servicio u oficina específica que trate temas de sostenibilidad urbana y sin embargo tienen que desarrollar proyectos de intervención urbana, que pueden contribuir de manera notable a la misma.

La primera fase de esta investigación, que se presenta a continuación, ha consistido en revisar las herramientas ya disponibles en el contexto europeo. La revisión ha permitido constatar las carencias e identificar principios y elementos claves para:

1 El proyecto de investigación toma como referencia las herramientas de apoyo al diseño urbano desarrolladas en la Cátedra Technical Ecology and Methods (TEAM) del Departamento de Urbanismo de la Universidad Técnica de Delft. Estas herramientas, que tienen un enfoque eminentemente académico, están compuestas por una serie de hojas de excel para ejecutar simulaciones numéricas del impacto de distintas opciones de diseño en relación con el soleamiento, viento, ruido, agua, elementos vegetales, trárico, suelo, ecología, densidad y distribución de zonas verdes (1)

2 A título orientativo, la Carta de Leipzig de 2007 recomienda hacer un mayor uso de los enfoques relacionados con la política integrada de desarrollo urbano; la Declaración de Toledo de 2010 destaca la idoneidad del enfoque integrado en las políticas de desarrollo urbano. para permitir la implicación de sus habitantes en dichas transformaciones. El barrio tipo alberga entre 5.000 y 15.000 habitantes y se extiende por una superficie de un diámetro máximo de alrededor de 800 metros, con distancias que no precisen desplazamientos de más de diez minutos andando. Esta dimensión es capaz de tolerar relaciones sociales más diversas que las del vecindario, en torno a asociaciones, actividades, equipamientos o instituciones y es un umbral que puede sostener niveles de servicios colectivos más complejizados (centros cívicos, biblioteca, educación secundaria, iglesia, centro de salud, mercado, comercio de especialización media, zonas verdes, etc.) (2).

\subsection{La perspectiva socioeconómica}

En Europa hay una creciente voluntad de acometer las intervenciones en barrios desde una perspectiva integral ${ }^{2}$. Esto implica que el diseño de las actuaciones y la planificación de las inversiones han de incorporar objetivos de revitalización del barrio que vayan más allá de la recualificación espacial. En la revisión se analiza cómo se consideran y se evalúan aspectos tales como cohesión social, estímulos a la economía local, condiciones de vida, participación y gobernanza.

Contar con herramientas de evaluación de la sostenibilidad urbana contribuiría a discernir si el cambio hacia planteamientos integrales se está produciendo realmente y si se obtienen los efectos esperados.

\subsection{Evaluación cuantitativa y cualitativa}

Incorporar durante la elaboración de propuestas de intervención urbana, procedimientos de evaluación tanto cualitativa como cuantitativa permitiría mejorar su impacto y eficacia. En la revisión se analiza si las herramientas disponibles permiten valorar diferentes opciones teniendo en cuenta criterios de sostenibilidad. En los últimos años ha habido un notable desarrollo de herramientas para evaluar la sostenibilidad -o más específicamente la eficiencia energética- en el sector de la edificación, como BREEAM, que no consideran la escala de barrio. LEED, que sí lo hace, se centra en la aplicación para nuevos desarrollos. Mientras tanto, distintas agencias, instituciones y gobiernos locales desarrollan herramientas de evaluación sobre sostenibilidad urbana ad hoc, con escasas posibilidades de poderse aplicar en otros lugares.

\section{METODOLOGIA}

La investigación se basa en el análisis exhaustivo de las herramientas de evaluación de la sostenibilidad urbana que cuentan con cierto reconocimiento en el contexto europeo. El 
análisis ha permitido contrastar qué herramientas hay disponibles y cómo se pueden adaptar para su aplicación en barrios de ciudades pequeñas y medias o en pueblos.

\subsection{Selección de herramientas objeto de análisis}

La mayor parte de las herramientas analizadas han sido desarrolladas por instituciones locales o nacionales en el marco de programas europeos de investigación financiados con fondos públicos. Al amparo de estos programas -uno de cuyos objetivos era la creación de Clústeres de conocimiento- se han ido sucediendo también proyectos y redes de difusión de experiencias y de buenas prácticas.

Uno de ellos, el proyecto PETUS (Practical Evaluation Tools for Urban Sustainability), liderado por la Universidad de Cardiff recopiló las herramientas de evaluación disponibles en el contexto europeo. Este conjunto de herramientas constituye el primer bloque analizado. El segundo bloque lo integran aquellas herramientas recopiladas en el proyecto CRISP (City Related Sustainability Indicators Project), que abordan temas relacionados con la regeneración urbana.

Tanto PETUS como CRISP se desarrollaron dentro del Quinto Programa Marco.

Se ha realizado una consulta exhaustiva a través de internet de dichos proyectos entre noviembre de 2009 y febrero de 2010. Esta revisión se complementa con el estudio posterior de proyectos incluidos en el Sexto programa marco, efectuado en mayo de 2011.

\subsection{Criterios de clasificación}

En los casos en los que es posible encontrar suficiente material relevante, se estudian y clasifican las herramientas según:

1) La escala a la que se aplica: edificio o manzana, barrio, ciudad y región o país.

2) La fase en la que se aplica, distinguiendo entre ex-ante o de diseño y ex-post o de monitorización, seguimiento o evaluación.

3) El objeto (O) al que se aplica la herramienta.

4) El tipo (T) de herramienta, distinguiendo entre guías o recomendaciones metodológicas, check-list, sistema de indicadores o modelos. Se indica a su vez si la herramienta trabaja con GIS, dado su enorme potencial futuro.

5) El agente (A) que la ha desarrollado, que puede ser público o privado, ligado a consultoras, instituciones europeas, centros de investigación o académicos o a gobiernos locales, regionales o nacionales.
6) La naturaleza de los temas tratados. En este apartado se distingue si la herramienta considera los aspectos físicos vinculados al metabolismo urbano (aire, suelo, agua, energía y clima, ruido, transporte, residuos, áreas naturales y zonas verdes, materiales y sistemas de construcción); si trata los aspectos sociales (comunidad, sentido del lugar o identidad, cohesión, gobernanza) o los económicos (empleo, gestión y desarrollo económico).

Los puntos del 1 al 5 se sintetizan en un cuadro resumen que se muestra en el apartado 3.2. El punto 6 se representa en el gráfico de la figura 1 del apartado 3.3, como una araña en la que aparecen todos los temas señalados anteriormente agrupados según su naturaleza. Cada tema recibe una puntuación en función del porcentaje de las herramientas que lo abordan. La tabla y el gráfico permiten visualizar la orientación y la importancia relativa que se da a cada tema.

\section{REVISION DE HERRAMIENTAS DE EVALUACIÓN}

\subsection{Hilos que se pierden}

Durante la fase de análisis de herramientas se chequearon 77 proyectos desarrollados dentro de la key Action 4 "Ciudad del mañana y patrimonio cultural" del Quinto Programa Maco (1998-2002). Estos proyectos se englobaban en la línea temática Integrating Cultural Assets into the Urban Setting. Lo primero que hay que remarcar es el alto porcentaje de proyectos que no son accesibles para el público en general, a pesar de que se desarrollaron con cargo a programas de investigación europeos ${ }^{3}$. Parecería razonable que proyectos financiados con fondos públicos sean accesibles y su información perdure a lo largo del tiempo, de manera que permitan seguir construyendo conocimiento e incluso que tengan una proyección futura alimentando nuevas aplicaciones o investigaciones. Sin embargo, cuando se realizó el análisis a principios de 2010, 33 de las 77 páginas de internet de los proyectos ya no estaban operativas, lo que supone el $43 \%$ del total. Es probable que en la actualidad el porcentaje haya incluso aumentado. Parecería que una vez acabado el proyecto -y la financiación- la línea no se continúa, los recursos y el conocimiento generado no se aprovechan y tampoco se puede valorar su pertinencia.

Por su parte el proyecto PETUS recogía dentro del epígrafe "Edificación y usos del suelo" 57 herramientas de evaluación de la sostenibilidad. Se ha podido estudiar casi la mitad de ellas, el 49\%. A través de los enlaces suministrados en la propia reseña de PETUS se puede acceder a información directa sobre 20 herramientas, en 8 los enlaces ya no fun-
${ }^{3}$ Es el caso por ejemplo del proyecto EUROGISE, dentro del programa TERRA, o del proyecto CORDIS Promoting Action for Sustainability Through Indicators at the Local Level in Europe (PASTILLE). 
${ }^{4}$ Al final del artículo se puede encontrar una explicación de los proyectos.

${ }^{5}$ Al final del documento se puede encontrar un listado de los Acrónimos con su respectivo significado. cionaban pero era posible encontrar material a través de una búsqueda específica. Algunas de estas herramientas no se han incorporado al estudio, bien por ser demasiado genéricas (comparación de países mediante el empleo de estadísticas nacionales o exposiciones conceptuales como enfoques multicriterio para toma de decisiones sin aplicación concreta), o demasiado específicas (por ejemplo matriz para seleccionar un emplazamiento en función de las condiciones del agua) o bien porque la documentación encontrada no permitía establecer con claridad su contenido ni su aplicación. En cuatro casos la información no es accesible al tratarse de herramientas internas o de consultoras privadas; dos plantean un problema con el idioma, pues solo ofrecen información en búlgaro; otras dos son instrumentos normativos (Código de Urbanismo de
Francia, evaluación de Impacto Ambiental de la UE) y no encajan en la aproximación del presente estudio De los proyectos en los que sí que se cuenta con información relevante, se han descartado aquellos cuyo objetivo principal es la creación de redes o difusión de buenas prácticas, aquellos proyectos centrados bien en el desarrollo de una tecnología o en la evaluación de una técnica o herramienta muy concreta -gestión de residuos, cibercoches, autobuses de hidrógeno-.

En el Sexto Programa Marco (2002-2006) la consideración de los temas urbanísticos pierde peso en favor de enfoques más sectoriales que dan prioridad a la eficiencia energética, al transporte y a la reducción de gases con efecto invernadero. Se analizaron STATUS, SENSOR, TISSUE y RAISE ${ }^{4}$.

Tabla 1

Clasificación de las herramientas según su ámbito de aplicación, aplicación y contenido ${ }^{5}$

\begin{tabular}{|c|c|c|c|c|}
\hline \multicolumn{5}{|c|}{ ESCALA DE APLICACIÓN } \\
\hline & Región/nación & Ciudad & Barrio & Edificio \\
\hline \multicolumn{5}{|c|}{ HERRAMIENTAS PARA LA FASE DE DISEÑO } \\
\hline $\mathrm{N}$ & ЕСОВОХХ & EUROGISE & BRE Checklist & ЕСОВОХХ \\
\hline $\mathrm{O}$ & Estrategia sectorial & & Nuevos desarrollos & Edificios existentes \\
\hline $\mathrm{T}$ & Modelo & GIS, Indicadores & Checklist +Benchmarks & Modelo \\
\hline A & Centro investigación & Agencia Local/Regional & Agencia púb+Consultoría & Centro investigación \\
\hline $\mathrm{N}$ & SLA & & COUNTRYSIDE & HQE Process \\
\hline $\mathrm{O}$ & Planes de desarrollo & & Interfaz urbano-rural & Nuevos o a rehabilitar \\
\hline $\mathrm{T}$ & Conceptos gobernanza & & Guía & Indicadores, métodos ev. \\
\hline A & Agencia Nacional & & University & Asociación empresas \\
\hline N & & & & MEMPD \\
\hline $\mathrm{O}$ & & & & Proyectos vivienda \\
\hline T & & & & Guía y Checklist \\
\hline A & & & & Inst. educ.+Asoc empresas \\
\hline N & & & & Sust. Checklist \\
\hline $\mathrm{O}$ & & & & Edifcio comunitario \\
\hline $\mathrm{T}$ & & & & Guía y Checklist \\
\hline A & & & & Red \\
\hline \multicolumn{5}{|c|}{ HERRAMIENTAS PARA LAS FASES DE SEGUIMIENTO Y EVALUACIÓN } \\
\hline $\mathrm{N}$ & CSD Framework & $\tilde{\mathrm{A}}-\mathrm{KOSTADT} 2000$ & & EMCP \\
\hline $\mathrm{O}$ & Política nacional sostenibilidad & Evaluación. Ecocity 2000 Project + Action Plans & & Gestión mantenim. \\
\hline $\mathrm{T}$ & Indicadores & Indicadores, objetivos & & Indicadores + benchmark \\
\hline A & Agencia NNUU & & & Inst. educ.+Asoc empresas \\
\hline N & HUD Framework & Moland & & \\
\hline $\mathrm{O}$ & Programas pobreza urbana & Dinámicas uso suelo & & \\
\hline $\mathrm{T}$ & GIS & GIS-Indicadores & & \\
\hline A & USA Agencia & Agencia Europea & & \\
\hline N & Spatial territorial indicators & ISTAT Environmental Indicators Set & & \\
\hline $\mathrm{O}$ & Políticas & Medio ambiente urbano & & \\
\hline $\mathrm{T}$ & GIS Indicadores & Indicadores & & \\
\hline A & Agencia Europea & Agencia nacional & & \\
\hline $\mathrm{N}$ & & PASTILLE & & \\
\hline $\mathrm{O}$ & & Ev. eficiencia política urbana & & \\
\hline $\mathrm{T}$ & & Guía + test & & \\
\hline A & & Universdades & & \\
\hline
\end{tabular}




\subsection{Evaluación ex-ante y ex-post}

Para la investigación las herramientas más relevantes son las que se aplican a barrios ya existentes, para su rehabilitación o revitalización con criterios sostenibles. Lamentablemente ninguna de las incluidas en PETUS que son accesibles públicamente, está expresamente diseñada para tal fin. En cualquier caso es interesante estudiar su enfoque, metodología, contenido y aplicación, de manera que se puedan extraer conclusiones sobre qué aspectos resultaría pertinente considerar en herramientas especificas para evaluar (también ex-ante) la sostenibilidad de intervenciones urbanas en barrios existentes.

Como se puede apreciar en la tabla 1 las herramientas elaboradas para ser aplicadas durante la fase de diseño tienen sobre todo un carácter orientativo, como guías y checklist. Por su parte prácticamente todas aquellas que se aplican para monitorizar o evaluar -y revisar- proyectos, programas y políticas, utilizan indicadores. Es cuando menos significativo que no se considere el empleo de indicadores para evaluar la sostenibilidad de la intervención durante la fase de concepción o diseño de la propuesta.

Se produce por otro lado una gradación de las herramientas disponibles según la escala de aplicación, las herramientas orientadas al diseño se centran en los edificios y, en menor medida en el barrio, mientras que las herramientas orientadas a la evaluación abordan las escalas superiores: la ciudad, áreas metropolitanas y programas y políticas regionales o nacionales. Hay efectivamente un déficit de herramientas de evaluación de la sostenibilidad urbana a escala de barrio.

En este punto cabe destacar que encontrar guías orientativas o metodológicas, checklists e indicadores no ha sido difícil. Sin embargo cuando se trata de modelos o de herramientas más complejas o más técnicas, con frecuencia solo se puede acceder a información derivada, es decir a informes o artículos sobre las mismas, no es posible su conocimiento directo. Hay excepciones, como por ejemplo el proyecto BUGS que está profusamente documentado.

Las guías y check-lists son herramientas útiles para ordenar el proceso de diseño y toma de decisiones, de manera que se tengan en cuenta los aspectos que en la guía se consideran relevantes. Sin embargo prácticamente en ningún caso se plantean como apoyo para la selección de alternativas. Pocas de las herramientas a aplicar en la escala de barrio han desarrollado indicadores. Se considera sin embargo que los que se emplean en varias de las herramientas para evaluar la sostenibilidad a escala de ciudad podrían ser adaptados para su consideración en la evaluación de intervenciones en barrios. Hay que contar sin embargo con las dificultades que existen para obtener datos desagregados y coincidentes con la delimitación del barrio o área de intervención. Este problema se da especialmente en el caso de los datos socioeconómicos; en cierta medida puede llegar a ser subsanado mediante el empleo de SIG, pero para buena parte del público objetivo, ésta no es una opción viable a corto plazo.

El proyecto GREENSCOM sugiere que, puesto que el urbanismo trata temas sociales que conllevan una gran complejidad inherente y dependen profundamente del contexto local, los programas de investigación deberían orientarse hacia la difusión de buenas prácticas y reuniones de intercambio de conocimiento. Entienden que no es realista generar un conjunto de herramientas que se puedan usar de mondo general (3). Sin embargo no parece oportuno renunciar a la posibilidad de medir, de anticipar y de evaluar en términos también cuantitativos las propuestas.

En este sentido herramientas de evaluación basadas en la comparación de alternativas ayudarían a urbanistas y planificadores a hacer explícitas las diferentes posibilidades. Pueden además contribuir a comunicar y discutir mejor las opciones con los distintos agentes y con la población. Si además se fijan valores de referencia sería posible comparar las metas ideales y posibles con los resultados reales a lo largo de la implementación.

En los proyectos de revitalización urbana hay factores clave que no pueden ser cuantificados. Por ejemplo si un proyecto plantea fomentar las actividades culturales como catalizadores de desarrollo económico ¿Cómo valorar su impacto? Si no es posible medirlo directamente como una relación de causa-efecto, al menos pueden establecerse relaciones con indicadores complementarios que hagan explícitas las hipótesis que sustentan el planteamiento y que permitan su contraste posterior. Estas herramientas facilitarían la replicabilidad de lecciones aprendidas, tanto en casos exitosos como en aquellos en los que no se alcanzaron los objetivos planteados.

\subsection{Los temas estrella}

La sostenibilidad tiene una triple dimensión: social, económica y ambiental. Sin embargo, si analizamos las herramientas de evaluación de la sostenibilidad urbana, que incorporan mecanismos para medirla, hay un gran desequilibrio en la consideración de estos tres aspectos. Como se puede apreciar en la 

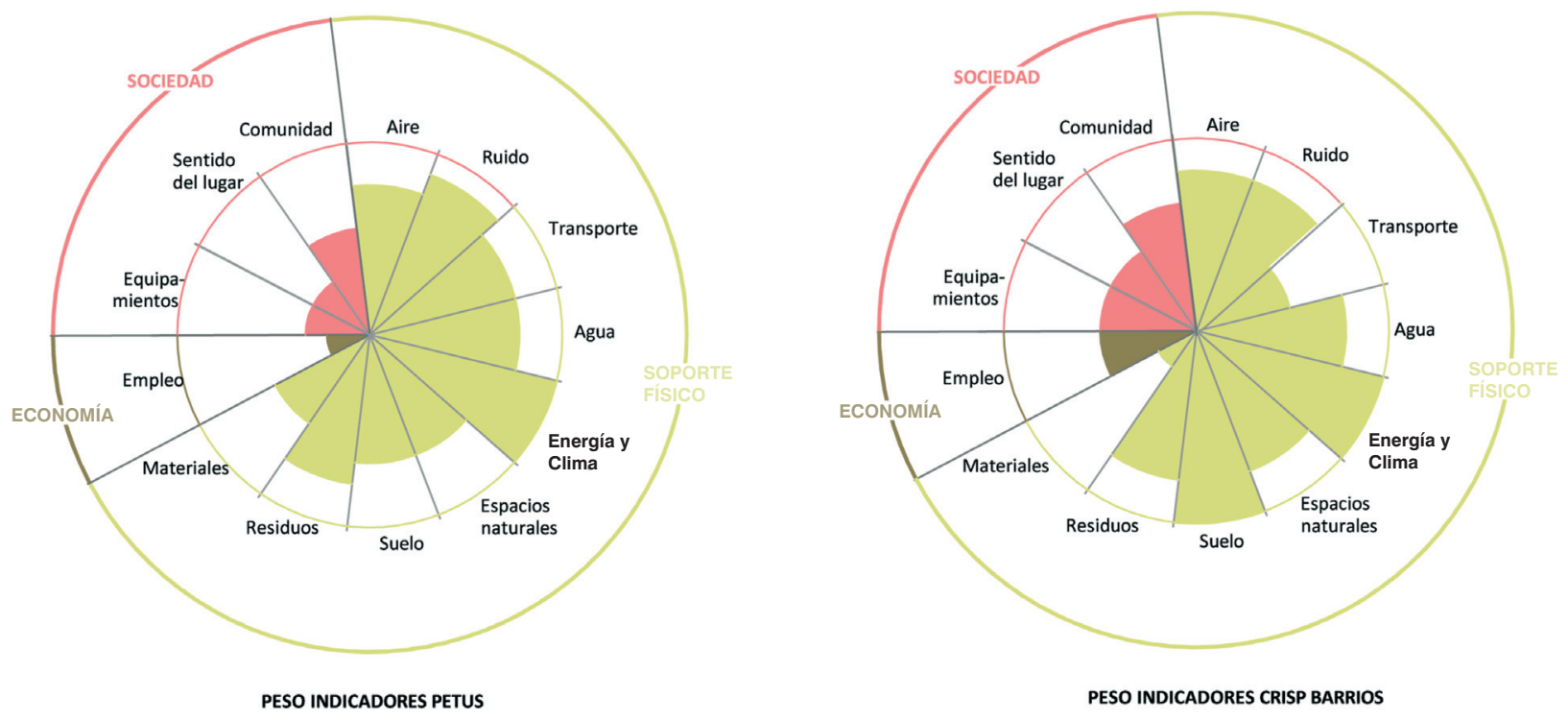

1. Soporte físico, sociedad y economía en las herramientas de evaluación de la sostenibilidad urbana ${ }^{6}$.

6 Los proyectos incluidos en PETUS que han sido analizados son los mismos que aparecen en la Tabla 1. Los proyectos incluidos en CRISP que han sido analizados son: INTEGAIRE, CLEANAIR, IANUS, LASALA21, BUGS, GREENSCOM, GREENSPACE， RUROS，URGE, PROPOLIS, SCATTER, TRANSPLUS, PROSPECTS, ISHTAR, ECOCITY, SELMA, ASI, FORTMED, REURBAN MOBIL, MASURIN, ECOPADEV, HQE2R, PRESCO, SHE, SUREURO, HOMESERVICES, ORMA， SAFEFLOOR, ARTISTS, CASTS, IRMA y STATUS. Los dos últimos corresponden al FP6. figura 1 el soporte físico -la sostenibilidad ambiental- es, con diferencia, el aspecto que más atención recibe. Esta dimensión incluye los temas relacionados con el aire, suelo, agua, energía y clima, ruido, transporte, residuos, áreas naturales y zonas verdes, materiales y sistemas de construcción). Es ciertamente más fácil medir y cuantificar la incidencia de una intervención urbana sobre el soporte físico y el flujo de materiales o de energía que medir su impacto social y económico, por su complejidad y porque se ven afectados en mayor medida por factores que exceden la intervención urbana.

En el segundo gráfico de la figura, se presentan las herramientas específicas de evaluación de intervenciones en barrios, recogidas por el proyecto CRISP, en donde los temas del soporte físico siguen teniendo un peso importante. En la ficha sobre cada herramienta que la red CRISP ha elaborado, la clasificación de los temas difiere respecto de la que se presenta aquí. Algunos temas como aire y ruido se clasifican como "indicadores sociales" pues afectan a la calidad de vida y a la salud de las personas. De alguna manera todos los temas incluidos en el soporte físico se relacionan con la calidad de vida. Por ello hemos considerado más oportuno reservar el concepto de sociedad para los que aluden a las interacciones sociales, y tanto el ruido como la calidad del aire se mantienen dentro del bloque de soporte físico.

Llama la atención cómo el tema de materiales ve reducir su presencia -puede deberse a que se asocie con la escala del edificio más que con la de barrio-. Lo mismo sucede con el transporte, en este caso tal vez porque su tratamiento se vincula a una escala superior, la de la ciudad o el área metropolitana. Dos temas aparecen en todas las herramientas: uno de ellos es el del cambio de usos de suelo, influido porque este ha sido un tema recurrente en los programas de la Unión Europea, por ejemplo con el proyecto Corine Land Cover. El segundo tema omnipresente es el de Energía y clima, lo cual deja patente que el cambio climático y la preocupación por la dependencia energética ocupa un lugar destacado en las agendas, no solo políticas sino también de las investigaciones. La mayoría de estos proyectos ponen énfasis en la eficiencia. En algunos se fijan indicadores y objetivos para limitar la cantidad de emisiones de $\mathrm{CO}_{2}$, reduciendo el impacto de las edificaciones en el cambio climático.

Aunque no estén representados en el gráfico, es interesante destacar que en estas herramientas para evaluar la intervención en barrios han aparecido otros temas como el de costes económicos (reducir consumos conduciría también a reducir costes), el de viviendas asequibles o el control de precios. Varios documentos destacan la importancia del modo de vida o incorporan objetivos de reducción del consumo y de la producción (4). El proyecto Äkostadt señala que los objetivos de desarrollo sostenible, de mejorar la salud y el bienestar de la sociedad y preservar la biodiversidad no se lograrán vía decreto. La articulación normativa, el establecimiento de límites y la prohibición de determinadas sustancias son necesarias, tan necesarias como lograr procesos creativos a escala local que refuercen los ecosistemas locales (5). 
Para avanzar en futuras propuestas se plantea diferenciar las transformaciones que afectan al medio físico de aquellas que implican cambios en el marco normativo y en el entorno cognitivo (6). Los cambios que afectan a aspectos no materiales como modificar la regulación o distribución de usos conllevan un menor consumo de recursos naturales que aquellos que implican la transformación de estructuras masivas.

\section{CONCLUSIONES Y PERSPECTIVAS}

\subsection{Dificultades para la aplicación y transferibilidad de herramientas de evaluación}

Faltan herramientas prácticas y accesibles para comparar y seleccionar alternativas de intervención en la escala de barrio, incorporando criterios de sostenibilidad en el proceso de toma de decisiones. El déficit es más acusado en la valoración de objetivos e impactos relacionados con los aspectos sociales y económicos.

Para avanzar en la gestación de herramientas apropiadas y apropiables, sería muy conveniente que -al menos-todos los proyectos de investigación financiados por fondos europeos en el marco de programas de desarrollo e intercambio de conocimiento, hicieran accesibles permanentemente sus resultados, no solo a través de informes o artículos, sino haciendo pública la propia herramienta. Sería interesante además que se basaran en programas de acceso universal y compatible, de manera que se pueda seguir construyendo sobre ellas. Ya existen potencialmente buenas herramientas de evaluación tanto ex ante como ex post que se ocupan de las zonas verdes (BUGS, GREENSPACE, RUROS o en menor medida, URGE), de la remodelación de edificios (SHE o SUREURO) del desarrollo de brownfields (RESCUE o SELMA) o de temas de tráfico (PROPOLIS). Las herramientas que se desarrollen para evaluar la sostenibilidad de las intervenciones podrían ligarse a ellas en lo que se refiere a los temas específicos de los que cada una se ocupa.

\subsection{Incorporar las variables sociales y económicas, un tema aún pendiente}

Las herramientas que pretenden ir más allá de medir la incidencia en el soporte físico se encuentran con la dificultad de enfrentarse a la complejidad del barrio como sistema social. Hasta ahora las herramientas de evaluación se han centrado sobre todo en el soporte físico y la sostenibilidad ambiental. Para ser coherentes con las declaraciones europeas que abogan por políticas urbanas integradas, la herramienta a desarrollar debería incorporar los aspectos sociales y económicos.
Por otro lado el desarrollo de una herramienta adecuada de evaluación ha de considerar las limitaciones inherentes a las intervenciones en entornos ya construidos. Parte de los condicionantes se derivan de las limitaciones legales, de la aceptación social, de la fragmentación de la propiedad, de la consideración de criterios de coste-eficacia y de la viabilidad técnica y económica.

\subsection{Herramientas prácticas}

Una de las principales limitaciones para aplicar herramientas de evaluación se deriva de la falta de datos adecuados. A menudo al trabajar en escalas menores, surge el problema de disponibilidad de datos desagregados, pues las estadísticas se suelen referir a escalas mayores o no coinciden con la delimitación del barrio. De todas formas se pueden identificar variables de medición y conexiones entre el barrio y la ciudad y entre el barrio y el edificio -como ilustra el proyecto IANUS-. Es recomendable valorar los impactos que la propuesta de intervención en el barrio tiene en las otras escalas, hacia arriba y hacia abajo.

Una última puntualización es necesaria. Al desarrollar una herramienta de evaluación hay que tener bien presente que debe ser útil y práctica. Ello implica evitar sistemas que se basan en enormes cantidades de tiempo y esfuerzo para recoger datos y cargarlos en el modelo. Las grandes ciudades o redes urbanas tienen departamentos específicamente dedicados a la sostenibilidad urbana. Pero no son las únicas que han de implicarse en el cambio hacia entornos urbanos más sostenibles. Las ciudades medianas y pequeñas e incluso los pueblos comparten una parte de la responsabilidad en el proceso y no disponen de grades presupuestos ni cuentan con suficientes equipos profesionales. Este grupo de ciudades y pueblos constituyen el público objetivo para el desarrollo de herramientas prácticas, útiles y de código abierto -y por tanto adaptables- que permitan evaluar la sostenibilidad de las intervenciones urbanas a escala de barrio.

\section{INFORMACION SOBRE ALGUNAS DE LAS HERRAMIENTAS ANALIZADAS}

\subsection{Herramientas de la base de datos de PETUS}

BRE Regional Sustainability Checklist for Developments. http://southeast.sustainabilitychecklist.co.uk/checklist. Una de las checklists se refiere específicamente al cambio climático. Cada pregunta ofrece una serie de opciones entre las que seleccionar la respuesta, algunas se refieren a cuestiones de diseño, otras a tecnologías e infraestructuras. 
CSD FRAMEWORK Commission on Sustainable Development Framework. http://www. un.org/esa/sustdev/natlinfo/indicators/indisd/ english/worklist.htm - Difícil de aplicar a la escala de barrios, maneja indicadores pensados para evaluar tendencias nacionales.

ECOBOXX. Interesante ejemplo de cómo se puede utilizar una herramienta de formulación de escenarios para definir la estrategia finlandesa de producción y consumo responsable. Lamentablemente no es posible acceder a la herramienta, la información ha desaparecido de la Institución que la desarrolló (VTT) y no responden a las consultas por correo electrónico.

EMCP Environmental Management Control Panel. Se refiere al monitoreo de edificios sobre aspectos relacionados con su mantenimiento. No tiene relación con el objetivo de la investigación.

European Common Indicators. http:// ec.europa.eu/environment/urban/common_ indicators.htm.

HQE PROCESS. High Environmental Quality. http://www.assohqe.org/.

HUD Housing and Urban Development. Publicaron un libro interesante sobre el empleo de sistemas de información geográfica para tratar la dimensión espacial de la vulnerabilidad urbana. http://www.nap.edu/ openbook/0309088747/html/.

ISTAT Environmental Indicators Set. Istituto nazionale di statistica Italia. Se trata de un conjunto de indicadores sobre los aspectos físicos basados en el enfoque conceptual de DPSIR (Fuerza Directriz-Presión-EstadoImpacto-Respuesta).

MEMPD. Manual on Environmental Management in Project Design. Un manual muy claro sobre la consideración de aspectos ambientales en el proceso de diseño. Se centra en viviendas, pero teniendo en cuenta los condicionantes y el tratamiento del entorno. Para cada posible impacto señala posibles soluciones. Documento a tener como referencia de herramienta práctica, amena y fácil de usar. http://www.europeangreencities.com/ pdf/publications/manualSustainableHP.pdf.

PASTILLE. Promoting Action for Sustainability at the Local Level in Europe.

\section{SLA. Sustainable Livelihoods Approach.}

\subsection{Herramientas del Quinto Programa Marco (no incluidas en PETUS)}

BUGS Benefits of Urban Green Space. http:// wwwa.vito.be/BUGS/. Incluye simulaciones de tráfico, ruido, calidad de aire a escala regional y micro. Algunas se basan en el programa de código abierto Envi-met. Tiene una visión integral, incluyendo urbanismo participativo. Muy bien documentado, se puede tomar como referencia para futuros trabajos.

PEGASUS Planning, Environment, Governance and Sustainability. La Guía que han producido se puede encontrar en http://www.Ine. be/themas/milieu-en-ruimtelijke-ordening/ pdfs/FinalReport_Pegasus.pdf. El proyecto consiste en evaluar la replicabilidad del enfoque neerlandés de desarrollo espacial y ambiental, a otros contextos europeos. Lo más interesante es la experiencia de "community of practice" de aprendizaje y la necesaria evolución desde una posición de "líderes expertos" al reconocimiento de la experiencia de otras ciudades. Sería aconsejable descubrir si se tradujo en cambios reales en las ciudades contraparte.

PLUS Participation, leadership and urban sustainability. Difícil de valorar pues solo se cuenta con una recopilación de los papers de una conferencia.

SELMA Spatial Deconcentration of Economic Land Use and Quality of Life in European Metropolitan Areas. http://selma.rtdproject. net/. El proyecto aborda una escala espacial superior pero aporta una estructura de indicadores y sistema de evaluación que puede alimentar desarrollos futuros en los aspectos de interrelación de la escala de barrio con escalas superiores.

SHE Sustainable Housing in Europe. http:// www.she.coop/.

\subsection{Herramientas del Sexto Programa Marco}

IRMA. Integrated Decontamination and Rehabilitation of Buildings, Structures and Materials in Urban Renewal. http://projweb. niras.dk/irma/index.php?id=645. Planteamiento ambicioso e interesante sobre cómo actuar en la renovación urbana reduciendo el consumo de recursos y la generación de residuos. Lamentablemente ni los informes parciales ni el final, ni el Código de Buenas Prácticas en sitios contaminados, ni el material de demostración y formación están colgados en la web.

STATUS, Sustainability Tools and Targets for the Urban Thematic Strategy. http://statustool.iclei.org/content.php/frontpage/?p=1. Incluye una herramienta de autoevaluación para gobiernos locales en las que se introducen las metas de sostenibilidad local y se obtiene una valoración de los logros alcan- 
zados. Incluye explicación clara sobre el establecimiento efectivo de metas, sobre el significado de cada ítem y sobre el cálculo de cada indicador.

TISSUE, Trends and indicators for monitoring the EU thematic strategy on sustainable development of urban environment. http:// cic.vtt.fi/projects/tissue/index2.html. Realiza una evaluación sistemática de los indicadores que están siendo empleados por distintas instituciones para monitorizar la estrategia europea de desarrollo sostenible del medio ambiente urbano y propone su propio set de indicadores, a tener en cuenta en el desarrollo de una herramienta de evaluación. Se centra en aspectos físicos, incorpora algunos indicadores sociales como el grado de satisfacción de la población o su participación en programas ambientales.

SENSOR, Sustainability Impact Assessment: Tools for Environmental, Social and Economic Effects of Multifunctional Land Use in European Regions. http://www.sensor-ip. eu/. Se aleja del objeto de la investigación y no tienen colgada ni la herramienta ni los resultados.

\section{REFERENCIAS}

(1) Jong, Taeke M. (ed.): Sun wind water earth life living, legends for design, p. 3, TU Delft, 2009. Excel exercises per chapter with VB applications available at http://team.bk.tudelft. $\mathrm{nl}$ (Publications Section).

(2) Alguacil, J.; Hernández, A.; Medina, M.; Moreno, C.: La ciudad de los Ciudadanos, p. 36, Ministerio de Fomento, Madrid, 1997.

(3) Aalbers, C. (ed.): GREENSCOM. WP 11 Evaluation of the GREENSCOM project and promotion of findings, p. 5, Alterra Green World Research Wageningen, the Netherlands, 2003.

(4) National Research Council: GIS for Housing and Urban Development. Committee on Review of Geographic Information Systems Research and Applications at HUD: Current Programs and Future Prospects. Washington, 2003.

(5) Graz Stadt Umweltamt: Eco-city 2000. Evaluation. Report (Expertise) of the Echo-Team of Graz, p. 8, 1999

(6) Simon Rojo, M.: "Urbanism and changing land use patterns". En Bruyns, Fuchs, Hoekstra, Meyer y van Nes (eds.): The European Tradition in Urbanism -and its Future. $4^{\text {th }}$ International Seminar on Urbanism and Urbanization, pp. 398-404. TU Delft, 2007

Se puede encontrar más información sobre la investigación y el desarrollo de la herramienta en www.surcosurbanos.org/tools.html. 